\title{
AN EFFECTIVE VERSION OF DILWORTH'S THEOREM
}

\author{
BY
}

HENRY A. KIERSTEAD

\begin{abstract}
We prove that if $\left(P,<^{P}\right)$ is a recursive partial order with finite width $w$, then $P$ can be covered by $\left(5^{w}-1\right) / 4$ recursive chains. For each $w$ we show that there is a recursive partial ordering of width $w$ that cannot be covered by $4(w-1)$ recursive chains.
\end{abstract}

0. Introduction. Loosely speaking, a subset $A$ of the natural numbers is recursive iff there exists an algorithm (i.e., a finite computer program) which upon input of a natural number $n$ outputs " 1 " if $n \in A$ and " 0 " otherwise. Similarly a partial ordering $(A, \leqslant)$ is recursive iff there is an algorithm which upon input of an ordered pair of natural numbers $(a, b)$ outputs " 1 " if $a \leqslant b$ and " 0 " otherwise. For a more careful definition of recursive relations see [R].

One of the attractions of finite combinatorics over infinite combinatorics is its explicit constructions. One never has to consider whether a finite object "really" exists. This paper is part of a program to enlarge the domain of finite combinatorics to certain infinite structures while preserving the explicit constructions of the smaller domain. We shall consider a recursive combinatorics whose domain is the recursive structures. Since finite structures are trivially recursive this domain does indeed extend that of finite combinatorics. Moreover each structure in this domain is explicitly exhibited by some finite computer program. Questions from the graph theory of this combinatorics have been studied by Bean [B], [B1], Kierstead [K], and Schmerl [S], [S1]. Generally their results relate the chromatic number of a recursive graph with certain properties to its recursive chromatic number.

The following example illustrates these ideas. Dilworth's theorem [D] asserts that any partial ordering of finite width $n$ can be covered by $n$ chains. If the partial ordering is finite, then one can actually exhibit these chains (by trial and error, if by no other method). The following easy argument demonstrates how Dilworth's theorem for countably infinite partial orderings follows from Dilworth's theorem for finite partial orderings.

Let $P=\left\{p_{i}: i \in N\right\}$. We show by induction that for all $i \in N$ there exist chains $C_{0}^{i}, \ldots, C_{n-1}^{i}$ such that:

(i) if $j<i$ and $k<n$ then $C_{k}^{j} \subset C_{k}^{i}$;

(ii) $p_{i} \in C_{0}^{i+1} \cup \ldots \cup C_{n-1}^{i+1}$;

(iii) if $Q$ is a finite subset of $P$ then $C_{0}^{i}, \ldots, C_{n-1}^{i}$ can be extended to chains that cover $Q$.

Received by the editors August 10, 1979 and, in revised form, June 17, 1980.

1980 Mathematics Subject Classification. Primary 03D45.

(c) 1981 American Mathematical Society 0002-9947/81/0000-0502/\$04.75 
For $j<n$ let $C_{j}^{0}=\varnothing$. Then (i) and (ii) are trivial and (iii) holds by Dilworth's theorem. Now suppose that we have $C_{0}^{j}, \ldots, C_{n-1}^{j}$ for all $j \leqslant i$. We show that there is an $m<n$ such that (i)-(iii) hold for $C_{0}^{i+1}, \ldots, C_{n-1}^{i+1}$ where

$$
C_{k}^{i+1}= \begin{cases}C_{k}^{i} & \text { if } k \neq m, \\ C_{k}^{i} \cup\left\{p_{i}\right\} & \text { if } k=m .\end{cases}
$$

Again (i) and (ii) are trivial. If (iii) did not hold for any $m<n$, then for each $m<n$ we could find a finite $Q_{m}$ to witness this. Then $Q=Q_{0} \cup \ldots \cup Q_{n-1}$ would witness that (iii) did not hold for $C_{0}^{i}, \ldots, C_{n-1}^{i}$. This is a contradiction. Clearly $\cup_{i \in N} C_{0}^{i}, \ldots, \cup_{i \in N} C_{n-1}^{i}$ are chains that cover $P$.

While the above argument shows that there is a cover of $P$ by $n$ chains it does not show how to effectively or computationally produce that cover. The problem is that at each stage $i$, in order to decide which chain to put $P_{i}$ into, we must perform the impossible task of considering each of the infinitely many finite subsets of $P$.

Schmerl showed that some noneffective steps would be required in any other proof by constructing a recursive width 2 partial ordering that cannot even be covered by 3 recursive chains. He asked whether there existed a finite bound, say $c$, on the number of recursive chains required to cover any recursive width $n$ partial ordering. In $\$ 1$ we show that for each finite $n$ there is indeed a finite $c$ and also that $D(n) \leqslant\left(5^{n}-1\right) / 4$, where $D(n)$ is the least such $c$ for $n$. Our proof also provides a single effective procedure which, when presented with any recursive partial ordering of finite width, say $n$, will cover it with $\left(5^{n}-1\right) / 4$ recursive chains. In $\$ 2$ we improve Schmerl's result by constructing a recursive width 2 partial ordering which cannot be covered by 4 recursive chains. Thus $\S \S 1$ and 2 show that $5<D(2) \leqslant 6$. In $\$ 3$ we prove a uniformity result concerning $D$. Finally in $\$ 4$ we pose some open questions.

Schmerl has shown that if $\left(P,<^{P}\right)$ is a recursive partial ordering of width $n$ which is recursively locally finite then $P$ can be covered by $n$ recursive chains. A result of the author in [K] identifies a class of recursive partial orderings of width $n$ that can be covered by $n+1$ recursive chains.

Finally we point out that our approach to recursive combinatorics differs from that of Jockush [J] and the approach of Metakides and Nerode [MN] to recursive algebra. When studying a noneffective result from infinite combinatorics Jockush analyzed its degree of noneffectiveness (unsolvability). Our approach is to modify the result so as to make it effective. Metakides and Nerode were interested in isolating those algebraic constructions that are effective. Thus their recursive algebra is a restriction of algebra in general. Our investigation of recursive combinatorics should be viewed as an extension of finite combinatorics to certain infinite structures. It is not a restriction of infinite combinatorics since it deals with essentially different questions.

Notation. Let $\left(P,<^{P}\right)$ be a partial ordering. A subset of $P$ consisting of pairwise comparable (incomparable) elements is called a chain (antichain). The width of $\left(P,<^{P}\right)$ is the least upper bound of the lengths of its antichains. If $a, b \in P$ and $a$ is incomparable to $b$ then we write $a \mid b$. If $a$ is comparable to $b$ we 
write $a<>b$. In this paper we shall be dealing with several different partial orderings. In $\S 1$ we shall define a new partial ordering $\left(A,<^{*}\right)$. $<^{*}$ will, of course, refer to this ordering. $<^{N}$ will refer to the usual order on the natural numbers. $\mid$ and $<>$ always refer to $\left(P,<^{P}\right)$. Words associated with partial orderings such as chain, successor, between, and maximum refer to $<^{P}$ unless appropriately modified. For example ${ }^{*}$-chain refers to $<^{*}$ and $N$-maximum refers to $<^{N}$. The usual distinctions are made between $<^{P},<^{*},<^{N}$ and $<^{P},<^{*}, \leqslant^{N}$.

$\left(P,<^{P}\right)$ is recursive iff $P$ is a recursive subset of $N$, the set of natural numbers, and $<^{P}$ is a recursive subset of $N \times N . B \cap\left\{i \in N: i<^{N} n\right\}$ will be denoted by $B^{n}$. Several times in this paper we will construct a set $B$ by induction. Thus at a certain point in the construction we shall know which of the first $n$ natural numbers are in $B$. At this point we could compute $B^{n}$ even though $B$ has not yet been defined. We shall slightly abuse notation by referring to $B^{n}$ before $B$ is completely defined. If $(C, R)$ is a binary relation then $(C, R)^{n}$ is $\left(C^{n}, R \mid C^{n} \times C^{n}\right)$. $\phi_{i}$ is the $i$ th partial recursive function of the appropriate number of variables as in [R]. $\phi_{i}^{(k)}(\bar{x})$ is $\phi_{i}(\bar{x})$ if the $i$ th Turing machine converges after at most $k$ steps in the computation and is undefined otherwise. The reader should be careful not to confuse $\phi_{i}^{s}$ and $\phi_{i}^{(s)}$.

The following definition is needed to state the uniformity results of $\S \S 1$ and 3. A total recursive function $f: N \times N \rightarrow N$ is a decision procedure for a partial ordering $\left(P,<^{P}\right)$ iff the following conditions hold.

(i) $f(x, x)=1$ iff $x \in P$.

(ii) If $x, y \in P$, then $f(x, y)=1$ iff $x \leqslant^{P} y$.

A partial recursive function $f: N \times N \rightarrow N$ is a decision procedure for $\left(P,<^{P}\right)$ on $A$ iff $P \subset A, f$ is total on $A \times A$ and (i) and (ii) hold whenever $x, y \in A$.

Finally we state two useful facts about partial orderings that will be used later. Let $\left(P,<^{P}\right)$ be a partial ordering and suppose that $p, q, r, p^{\prime}, q^{\prime} \in P$.

0.1 . FACT. If $p<^{P} q, p\left|p^{\prime}, q\right| q^{\prime}$, and $q^{\prime}<^{P} p^{\prime}$, then $p \mid q^{\prime}$ and $q \mid p^{\prime}$.

0.2. FACT. If $p<^{P} q<^{P} r, p \mid p^{\prime}$ and $r \mid p^{\prime}$ then $q \mid p^{\prime}$.

1. An upper bound for $D$. For the purpose of exposition we present three increasingly stronger theorems in this section. The last is the main result. The first is a very special case of little interest except that it motivates the method of proof used in the much more complex general case. The second is the main result restricted to the width 2 case. It is also a special case of the inductive step used to prove the most general result.

1.1. TheOREM. Let $\left(P,<^{P}\right)$ be a width 2 recursive partial ordering. If $P$ contains a recursive chain $B$ such that for every $p \in P-B$ there exists a unique $p^{\prime} \in B$ such that $p \mid p^{\prime}$, then $P$ can be covered by 4 recursive chains.

Proof. It suffices to show that $A=P-B$ can be covered by 3 recursive chains. For this purpose it is useful to add more structure to $A$.

1.2. Definition. For $p, q \in A, p<^{*} q$ iff at least one of the following holds:

(1) $p<^{P} q$;

(2) there exist $p^{\prime}, q^{\prime} \in B$ such that $p^{\prime}<^{P} q^{\prime}, p^{\prime} \mid p$, and $q^{\prime} \mid q$. 
By the conditions on $B$ and the width of $\left(P,<^{P}\right)$ it is easy to see that $\left(A,<^{*}\right)$ is a recursive linear ordering.

1.3. Definition. For $p, q \in A, p \sim q$ iff there exists $p^{\prime} \in B$ such that $p \mid p^{\prime}$ and $p^{\prime} \mid q$.

Clearly $(A, \sim)$ is a recursive equivalence relation. The following lemma provides the necessary combinatorial information for developing an algorithm to cover $A$ by recursive chains.

1.4. LemMA. (i) Each $\sim$ class is convex relative to $\left(A,<^{*}\right)$, i.e., if $p \sim r$ and $p \leqslant{ }^{*} q \leqslant{ }^{*} r$ then $p \sim q$.

(ii) Each class is a chain.

(iii) If $p<^{*} q<^{*} r$ and $p \nsim q \nsim r$ then $p<^{P} r$.

Proof. (i) This is just a restatement of Fact 0.2 .

(ii) This follows from the width of $\left(P,<^{P}\right)$.

(iii) Let $q^{\prime} \in B$ be such that $q \mid q^{\prime}$. Using the definitions of $\left(A,<^{*}\right)$ and $(A, \sim)$ it follows that $p<^{P} q^{\prime}<^{P} r$.

To finish the proof of Theorem 1.1 we provide an algorithm for covering $A$ by 3 recursive chains.

Let $q^{-}={ }^{*}$-max $\left\{p \in A^{q}: p<^{*} q\right\}$ and let $q^{+}={ }^{*}$-min $\left\{p \in A^{q}: q<{ }^{*} p\right\}$. At stage $n+1$ if $n \in A$ we shall decide which of 3 sets $E_{0}, E_{1}, E_{2}$ to put $n$ into. We shall do this in such a way that we preserve the following conditions:

(3) if $p, r \in A^{n+1}$ and $p \sim r$ then $p$ and $r$ both belong to the same $E_{i}$;

(4) if $p, r \in E_{i}^{n+1}$ and $p \nsim r$ then there exists $q \in A^{n+1}$ such that $q$ is *-between $p$ and $r$ and $p \nsim q \nsim r$.

So if there exists $p \in A^{n+1}$ such that $p \sim n$ and $p \in E_{i}$, put $n$ into $E_{i}$. Otherwise put $n$ into $E_{j}$, where $j$ is the least $k$ such that $q^{-}, q^{+} \notin E_{k}$. This is possible because we have $3 E_{i}$ to choose from. We must check that (3) and (4) are preserved and that each $E_{i}$ is a chain. The first case of the algorithm assures that (3) will continue to hold. Consider (4). Case 1: $n \sim p$ and $p \in E_{i}^{n}$. Then $n \in E_{i}$. Suppose $r \in E_{i}^{n}$ and $n \nsim r$. Then $r \propto p$ and by the inductive hypothesis there exists $s$ such that $s$ is *-between $p$ and $r$ and $p \nsim s \nsim r$. By the convexity of $\sim, s$ is *-between $n$ and $r$. Case 2: $n \nsim p$ for any $p \in A^{n}$. Say $n \in E_{j}$. Suppose $r \in E_{j}^{n}$. Without loss of generality assume that $n<^{*} r$. Then $n<^{*} n^{+}<^{*} r$ and $n \propto n^{+}$. Since the algorithm put $n$ in $E_{i}, n^{+} \notin E_{i}$. Thus by the inductive hypothesis $n^{+} \nsim r$. Finally by (4) and Lemma 1.4(ii), (iii) each $E_{i}^{n}$ is a chain. Thus each $E_{i}$ is a chain.

We shall use the same basic approach to prove the next two theorems. However, as the setting becomes progressively more general, the definitions of $\left(A,<^{*}\right)$ and $(A, \sim)$ will become progressively more complicated. Also the basic combinatorial lemma will become weaker and more difficult to prove.

\subsection{TheOREM. $D(2) \leqslant 6$.}

Proof. Let $\left(P,<^{P}\right)$ be a recursive partial ordering of width 2 . We begin by constructing a recursive chain $B$ which resembles the $B$ of Theorem 1.1 as nearly as possible. Let $B=\left\{p \in P\right.$ : for all $\left.q \in B^{p}, q<>p\right\}$. We shall cover $A=P-B$ 
by 5 recursive chains. Notice that each element of $A$ is incomparable to at least one, but possibly more than one, element of $B$. This necessitates changes in the definitions of $\left(A,<^{*}\right)$ and $(A, \sim)$. Define $q^{-}$and $q^{+}$as before, but with respect to the new $A$ and $B$.

1.6. Definition. For $p, q \in A, p<^{*} q\left(q<^{*} p\right)$ iff at least one of the following holds:

(1) $p<^{P} q\left(q<^{P} p\right)$;

(2) for all $p^{\prime}, q^{\prime} \in B$, if $p^{\prime} \mid p$ and $q^{\prime} \mid q$ then $p^{\prime}<^{P} q\left(q^{\prime}<^{P} p^{\prime}\right)$.

Notice that because the width of $\left(P,<^{P}\right)=2$, if clause (1) does not apply to $p$ and $q$ and there exist $p^{\prime}, q^{\prime} \in B$ such that $p^{\prime}\left|p, q^{\prime}\right| q$, and $p^{\prime}<^{P} q^{\prime}$, then for all $\hat{p}, \hat{q} \in B, \hat{p} \mid p$ and $\hat{q} \mid q$ implies that $\hat{p}<^{P} \hat{q}$. With this remark we leave it to the reader to check that $\left(A,<^{*}\right)$ is a recursive linear ordering.

The following facts are easily checked.

$0.1^{*}$. FACT. If $p, q \in A, p^{\prime}, q^{\prime} \in B, p\left|p^{\prime}, q\right| q^{\prime}, p<^{*} q$, and $q^{\prime}<^{P} p^{\prime}$, then $p \mid q^{\prime}$ and $q \mid p^{\prime}$.

0.2*. FACT. If $p, q, r \in A, s^{\prime} \in B, p\left|s^{\prime}, r\right| s^{\prime}$, and $p<^{*} q<^{*} r$, then $q \mid s^{\prime}$.

1.7. Definition. We define $(A, \sim)$ by specifying $(A, \sim)^{q}$ for each $q \in N$. Suppose inductively that $(A, \sim)^{q}$ has been defined and $q \in A$. For $p \in A^{q+1}$, let $p \sim q$ and $q \sim p$ iff at least one of the following holds:

(3) $p=q$;

(4) $p \sim q^{-}$and for some $q^{\prime} \in B^{q}, q^{-} \mid q^{\prime}$ and $q^{\prime} \mid q$;

(5) not (4), $p \sim q^{+}$, and for some $q^{\prime} \in B^{q}, q \mid q^{\prime}$ and $q^{\prime} \mid q^{+}$.

We would like to have made $p \sim q$ iff there existed $q^{\prime} \in B^{q}$ such that $p \mid q^{\prime}$ and $q^{\prime} \mid q$. Using Fact 0.2 , it is easily seen that a new equivalence class is created at stage $q+1$ iff there do not exist $p \in A^{q}$ and $q^{\prime} \in B^{q}$ such that $p \mid q^{\prime}$ and $q^{\prime} \mid q$. However, it is possible that at stage $q+1$ we would like to make $q$ equivalent to two inequivalent elements of $A^{q+1}$. In this case the definition is biased towards the smaller of the two elements. Also, if we make $q \sim p$ and $p \sim r$ we are forced to make $q \sim r$ even if $q$ and $r$ do not share an incomparable element in $B^{q}$. With these remarks it is easy to check that $(A, \sim)$ is a recursive equivalence relation.

The following proposition is needed for the basic combinatorial lemma.

1.8. Proposition. If $p, q \in A^{n+1}, p \sim q$, and $q$ is the *-immediate successor of $p$ in $A^{n+1}$, i.e., $q=^{*}$-min $\left\{r \in A^{n+1}: p<^{*} r\right\}$, then there exists $p^{\prime} \in B^{n+1}$ such that $p \mid p^{\prime}$ and $p^{\prime} \mid q$.

Proof. We argue by induction on $n$. By the inductive hypothesis we may assume that $p=n$ or $q=n$. The argument is similar in both cases so assume that $p=n$. If $p \sim q$ by (5) we are done since $p^{+}=q$. Suppose $p \sim q$ by (4). Then $q \sim p^{-}$. Also $q$ is the *-immediate successor of $p^{-}$in $A^{n}$. Thus by the inductive hypothesis there exists $p^{\prime} \in B^{n} \subset B^{n+1}$ such that $p^{-} \mid p^{\prime}$ and $p^{\prime} \mid q$. Thus by Fact $0.2^{*}, p \mid p^{\prime}$.

1.9. LEMMA (BASIC COMBINATORIAL LEMMA). (i) Each $\sim$ class is convex.

(ii) Each class is a chain.

(iii) If $p, q, r, s \in A, p<^{*} q<^{*} r<^{*} s$ and $p \propto q \nsim r \propto s$ then $p<^{P} s$. 
Proof. (i) Suppose $p<^{*} q<^{*} r$ and $p \sim r$. We shall show by induction on $m=N-\max \{p, q, r\}$ that $q \sim r$. Case 1: $m=p$. Then $p^{-}<^{*} p<^{*} p^{+}<^{*} q$ $<^{*} r$ and $p^{-} \sim r$ or $p^{+} \sim r$. In either case the inductive hypothesis shows that $q \sim r$. Case 2: $m=q . p \leqslant^{*} q^{-}<^{*} q<^{*} q^{+} \leqslant{ }^{*} r$. By the inductive hypothesis $q^{-} \sim r \sim q^{+}$. Also $q^{+}$is the ${ }^{*}$-immediate successor of $q^{-}$in $A^{q}$. Thus there exists $q^{\prime} \in B^{q}$ such that $q^{-} \mid q^{\prime}$ and $q^{\prime} \mid q^{+}$. By Fact $0.2^{*}, q \mid q^{\prime}$. Thus $q \sim r$ by (4). Case 3: $m=r$. The argument is similar to Case 1 .

(ii) Suppose $p \sim q$ and $p<^{*} q$. Let $m=N-\max \{p, q\}$. We shall show by induction on the number of elements of $A^{m *}$-between $p$ and $q$ that $p<^{P} q$. If $q$ is the ${ }^{*}$-immediate successor of $p$ in $A^{m}$ then by Proposition 1.8 there exists $p^{\prime} \in A^{m}$ such that $p \mid p^{\prime}$ and $p^{\prime} \mid q$. Thus $p<^{*} q$ by (1), i.e., $p<^{P} q$. If $q$ is not the ${ }^{*}$-immediate successor of $p$ in $A^{m}$ choose $r \in A^{m}$ such that $p<^{*} r<{ }^{*} q$. By (i) $p \sim r \sim q$. Thus by the inductive hypothesis $p<^{P} r<^{P} q$.

(iii) First we prove two claims.

Claim 1. If $r, s \in A, r \nsim s, r<^{*} s, r^{\prime} \in B^{r}, r \mid r^{\prime}$ and $r$ is the initial (i.e., $N$-least) member of its $\sim$ class then $r^{\prime}<P$.

Proof. First we show that if $t \in A, r<^{*} t$, and $r^{\prime} \mid t$ then $r \sim t$. We argue by induction on the number of elements of $A^{t}{ }^{*}$-between $r$ and $t . r<^{N} t$ since otherwise $r$ is not the initial element of its $\sim$ class. By Fact $0.2^{*}, t^{-} \mid r^{\prime}$. Thus by the inductive hypothesis $r \sim t^{-}$. By (4) $r \sim t$. Since $r \nsim s$ it must be that $r^{\prime}<>s$. Let $s^{\prime} \in B^{s}$ be such that $s^{\prime} \mid s$. Not $s^{\prime}<^{P} r^{\prime}$ since otherwise by Fact $0.1^{*}, s \mid r^{\prime}$. Thus $r^{\prime}<^{P} s^{\prime}$ and not $s<^{P} r^{\prime}$. The only possibility is that $r^{\prime}<^{P} s$.

Claim 2. If $q, r \in A, q<^{*} r, q \nsim r, r^{\prime} \in B^{r}, r^{\prime} \mid r$ and both $q$ and $r$ are the initial elements of their respective $\sim$ classes then $q<^{P} r^{\prime}$.

Proof. $q<>r^{\prime}$ since otherwise the $N$-max $\{p, q\}$ would not be the initial element of its $\sim$ class. Let $q^{\prime} \in B^{q}$ be such that $q^{\prime} \mid q$. Not $r^{\prime}<^{P} q^{\prime}$ since otherwise by Fact $0.1^{*}, q \mid r^{\prime}$. Thus $q^{\prime}<^{P} r^{\prime}$ and not $r^{\prime}<^{P} q$. The only possibility is that $q<^{P} r^{\prime}$.

Now we continue the proof of (iii). Without loss of generality we may assume that $q$ and $r$ are the initial elements of their equivalence classes. Let $r^{\prime} \in B^{r}$ be such that $r^{\prime} \mid r$. Then $r^{\prime}<^{P} s$ by Claim 1 and $q<^{P} r^{\prime}$ by Claim 2. Thus $q<^{P} s$. Let $q^{\prime} \in B^{q}$ be such that $q^{\prime} \mid q$. Clearly $q^{\prime}<^{P} r^{\prime}<^{P} s$. Since the width of $\left(P,<^{P}\right)$ is 2 , $p<>q$ or $p<>q^{\prime}$. In either case it is easy to verify that $p<^{P} s$.

To finish the proof of Theorem 1.5 we provide an algorithm for covering $A$ by 5 recursive chains. At stage $n+1$ if $n \in A$ we shall decide which of 5 sets $E_{0}, \ldots, E_{4}$ to put $n$ into. We shall do this in such a way that we preserve the following conditions:

(6) if $p, s \in A^{n+1}$ and $p \sim s$ then $p$ and $s$ both belong to the same $E_{i}$;

(7) if $p, s \in E_{i}^{n+1}$ and $p \nsim s$ then there exist $q, r \in A^{n+1}$ such that $q$ and $r$ are *-between $p$ and $s, q \nsim r$ and neither $q$ nor $r$ is equivalent to either $p$ or $s$.

So if there exists $p \in A^{n+1}$ such that $p \sim n$ and $p \in E_{i}$ put $n$ into $E_{i}$. Otherwise put $n$ into $E_{j}$ where $j$ is the least $k$ such that no element of the two $\sim$ classes *-immediately preceding the $\sim$ class of $n$ in $A^{n+1}$ and no element of the two $\sim$ classes *-immediately succeeding the $\sim$ class of $n$ in $A^{n+1}$ is in $E_{k}$. This is possible 
by (6) and the fact that there are $5 E_{i}$ to choose from. We leave it to the reader, using Lemma 1.9, to check that (6) and (7) are preserved and that each $E_{i}$ is a chain.

1.10. TheOREM. $D(n) \leqslant{ }^{N}\left(5^{n}-1\right) / 4$.

Proof. Clearly $D(1)=1$. Thus it suffices to show that $D(n+1)<{ }^{N} 1+5 D(n)$. Notice that this is exactly what we did in the width 2 case. Let $\left(P,<^{P}\right)$ be a recursive partial ordering of width $n+1$. Define $A$ and $B$ as in the previous theorem. We shall show that $D(n+1) \leqslant{ }^{N} 1+5 D(n)$ by constructing a recursive partial ordering $\left(A,<^{*}\right)$ such that:

(1) the width of $\left(A,<^{*}\right)$ is $N$-less than $n+1$;

(2) every recursive *-chain can be covered by 5 recursive $P$-chains.

These are just generalizations of the properties of $\left(A,<^{*}\right)$ in the width 2 case.

1.11. Definition. We define $\left(A,<^{*}\right)$ by specifying $\left(A,<^{*}\right)^{q}$ for each $q \in N$. Suppose inductively that $\left(A,<^{*}\right)^{q}$ has been defined and $q \in A$. For $p \in A^{q+1}$, let $p<{ }^{*} q\left(q<{ }^{*} p\right)$ iff at least one of the following holds:

(3) $p<^{P} q\left(q<^{P} p\right)$;

(4) for all $p^{\prime}, q^{\prime} \in B^{q}$, if $p^{\prime} \mid p$ and $q^{\prime} \mid q$ then $p^{\prime}<^{P} q^{\prime}\left(q^{\prime}<^{P} p^{\prime}\right)$;

(5) there exists $r \in A^{q}$ such that $p<^{*} r\left(r<^{*} p\right)$ and (3) or (4) holds when $p$ is replaced by $r$.

We would like to have defined $\left(A,<^{*}\right)$ as in the width 2 case. However when the width is greater than 2 that definition may not yield a recursive relation. To remedy this situation we are forced to bound the quantifier in (4). But this produces a relation which may not be transitive. Thus we are forced to add (5). Fortunately, it turns out that in (5) we need only search over $A^{q}$ to obtain the appropriate transitive closure. The following lemma completes the proof of the theorem.

1.12. LEMMA. $\left(A,<^{*}\right)$ is a recursive partial ordering which satisfies (1) and (2).

Proof. Clearly $\left(A,<^{*}\right)$ is recursive. We shall show that $\left(A,<^{*}\right)$ is a partial ordering by showing that for every $n \in N,\left(A,<^{*}\right)^{n}$ is a partial ordering. We argue by induction on $n$. Assume $\left(A,<^{*}\right)^{n}$ is a partial ordering and consider $\left(A,<^{*}\right)^{n+1}$.

Transitivity. Suppose $p, q, r \in A^{n+1}, p<^{*} q$, and $q<^{*} r$. We must show that $p<^{*} r$. If $q \neq n$ then $p<^{*} r$ by the inductive hypothesis or (5). So assume $q=n$. Choose $p_{0}, r_{0} \in A^{n}$ so that $p \leqslant{ }^{*} p_{0}<^{*} q<^{*} r_{0}<{ }^{*} r$ and in $A^{n}$ there are no elements *-between $p_{0}$ and $q$ or $q$ and $r_{0}$. By the transitivity of $\left(A,<^{*}\right)^{n}$ it suffices to show that $p_{0}<{ }^{*} r_{0}$.

By our choice of $p_{0}$ we know that $p_{0}<{ }^{*} q$ by (3) or (4), but not by (5). Similarly $q<^{*} r_{0}$ by (3) or (4). Case 1: $p_{0}<^{*} q$ by (3) and $q<{ }^{*} r_{0}$ by (3). Then by the transitivity of $\left(P,<^{P}\right), p_{0}<^{*} r_{0}$ by (3). Case $2: p_{0}<{ }^{*} q$ by (4) and $q<{ }^{*} r_{0}$ by (4). Using the transitivity of $\left(P,<^{P}\right)$ again we see that $p_{0}<{ }^{*} r_{0}$ by (4). Case 3: $p_{0}<^{*} q$ by (3) and $q<{ }^{*} r_{0}$ by (4). Suppose $p_{0}^{\prime}, q^{\prime} \in B, p_{0} \mid p_{0}^{\prime}$ and $q \mid q^{\prime}$. By Fact 0.1 , $p_{0}^{\prime}<^{P} q^{\prime}$ or $p_{0}^{\prime} \mid q$. Thus $p_{0}<^{*} r_{0}$ by (4). Case 4: $p_{0}<{ }^{*} q$ by (4) and $q<{ }^{*} r_{0}$ by (3). The argument is similar to Case 3. 
Antireflexivity. Suppose there exists $q \in A^{n+1}$ such that $q<^{*} q$. This cannot be by (3) or (4). Thus by (5) there exists $r \in A^{q}$ such that $q<^{*} r<^{*} q$. But then $r<^{*} q<^{*} r$ and, by the transitivity of $\left(A,<^{*}\right)^{n+1}, r<^{*} r$. This contradicts the antireflexivity of $\left(A,<^{*}\right)^{n}$.

To prove that the width of $\left(A,<^{*}\right)$ is less than $n+1$ it suffices to show that if $I$ is a *antichain then there exists $b \in B$ such that $I \cup\{b\}$ is a $P$-antichain. We argue by induction on the cardinality of $I$. If the cardinality of $I$ is 1 then we are done by the definition of $A$ and $B$. So let $i_{0}$ and $i_{1}$ be distinct members of $I$. By the inductive hypothesis there exist $b_{0}, b_{1} \in B$ such that $\left\{b_{0}\right\} \cup\left(I-\left\{i_{0}\right\}\right)$ and $\left\{b_{1}\right\} \cup$ $\left(I-\left\{i_{1}\right\}\right)$ are $P$-antichains. Say $b_{0} \leqslant^{P} b_{1}$. If $i_{0} \mid b_{0}$ or $i_{1} \mid b_{1}$ we are done, so suppose not. Then $b_{0}<^{P} i_{0}$ and $i_{1}<^{P} b_{1}$. Since not $i_{0}<{ }^{*} i_{1}$ by (4) and Fact 0.2 there exists $b \in B$ such that $i_{0} \mid b$ and $b \mid i_{1}$. Clearly $b_{0}<^{P} b<^{P} b_{1}$. For every $j \in I \backslash\left\{i_{0}, i_{1}\right\}, j \mid b_{0}$ and $j \mid b_{1}$. Thus using Fact $0.2,\{b\} \cup I$ is a $P$-antichain.

Finally we show that every recursive ${ }^{*}$-chain $C$ can be covered by 5 recursive $P$-chains. Define an equivalence relation $(C, \sim)$ as in Definition 1.7 but with $A$ replaced by $C$. Then Lemma 1.9 still holds when $(A, \sim)$ is replaced by $(C, \sim)$. The proof is the same except that a little more care is needed to justify the next to last line. With this established we can use the same algorithm as before (relativized to $(C, \sim))$ to produce a covering of $C$ by 5 recursive $P$-chains.

For each $n \in N$ the proof of Theorem 1.10 provides an algorithm which, when applied to any effectively given partial ordering of width $n$, will produce a covering of the domain of that partial ordering by at most $\left(5^{n}-1\right) / 4$ recursive chains. The algorithm consists of nesting the inductive proof of Theorem $1.10 n-1$ times during an effective enumeration of the partial ordering. Moreover, when one considers the procedure carefully it is apparent that it is not necessary to know the depth of the nesting beforehand. Hence we can devise an algorithm which, when applied to any effectively given partial ordering, will produce a cover of the domain of that partial ordering by recursive chains. Furthermore if the partial ordering has finite width, say $w$, then this algorithm will cover its domain by, at most, $\left(5^{w}-1\right) / 4$ recursive chains. The result discussed in this paragraph may be stated in the language of recursion theory as follows.

1.13. Corollary. There is a recursive function $f$ such that if $e$ is the index of $a$ decision procedure for a binary relation, $\left(P,<^{P}\right)$, which is a partial ordering, then $f(e)$ is the index of a decision procedure for a binary relation $E$ such that if we let $S_{i}=\{x$ : $(x, i) \in E\}$, then each $S_{i}$ is a chain and each $p \in P$ belongs to some set $S_{i}$. Furthermore if $\left(P,<^{P}\right)$ has width $w$, then at most $\left(5^{w}-1\right) / 4$ of the sets $S_{i}$ are nonempty.

1.14. REMARK. Consider the following naive algorithm for covering an effectively given partial ordering $\left(P,<^{P}\right)$ by recursive chains $C_{0}, C_{1}, \ldots$ Suppose $P=\left\{p_{i}\right.$ : $i \in N\}$. Put $p_{0}$ into $C_{0}$. Now suppose that we have assigned each $p_{j}$, for $j<^{N} i$, to some $C_{k}$. Put $p_{i}$ into $C_{m}$, where $m$ is the first natural number such that $p_{i}$ is comparable to all the $p_{j}$ already in $C_{m}$. It is not difficult to find a recursive width 2 partial ordering which the above algorithm could not cover by finitely many chains. 
2. A lower bound for $D$. In order to find lower bounds for $D$ it is useful to consider the following game. The main results of $\$ \$ 1$ and 2 could have been phrased in terms of this game instead of in terms of recursive partial orderings and recursive chains.

2.1. Definition. Let $G(m, n)$ be the following infinite game for two players $X$ and $Y . X$ plays first. At his $(k+1)$ st turn $X$ will have constructed a finite partial ordering of width at most $n$. $Y$ will have covered this partial ordering by $m$ sets. $X$ makes his $(k+1)$ st play by extending his partial ordering to one new element in such a way that the width is still at most $n$. $Y$ then makes his $(k+1)$ st play by adding this new element to one of the sets in his covering. If at the end of $\omega$ plays each of the $m$ sets in $Y$ 's cover is a chain, $Y$ wins; otherwise $X$ wins. At times we may refer to $Y$ 's moves as coloring a point.

An easy result from game theory shows that for any $n$ and $m$ either $X$ or $Y$ must have a winning strategy for the game $G(m, n)$. The alert reader will notice that the proof of Theorem 1.1 actually shows that if $\left(5^{n}-1\right) / 4 \leqslant^{N} m$ then $Y$ has a winning strategy for $G(m, n)$. This motivates the following definition.

2.2. Definition. $D^{\prime}$ is the function defined on the positive natural numbers such that $D^{\prime}(n)$ is the least natural number $m$ for which $Y$ has a winning strategy for $G(m, n)$.

\subsection{LEMMA. $D^{\prime}(n) \leqslant^{N} D(n)$ for all $n \in N$.}

Proof. We shall show that if $m<^{N} D^{\prime}(n)$ then $m<^{N} D(n)$. So suppose $m$ $<^{N} D^{\prime}(n)$. Then $X$ has a winning strategy, say $S$, for $G(m, n)$. Moreover it is easy to see that there is a fixed finite $s$ such that, no matter how $Y$ plays, if $X$ follows $S$ then $X$ will be assured of a win after $s$ plays. Since a finite initial segment of $S$ assures a win, we may assume that $S$ is recursive. To show that $m<^{N} D(n)$ we must construct a recursive width $n$ partial ordering, say $\left(P,<^{P}\right)$, that cannot be covered by $m$ recursive chains. Equivalently, we must construct $\left(P,<^{P}\right)$ so that for all $\phi$ :

(1) if $\phi$ is a partial recursive function defined on $P$ and the range of $\phi$ is $\{0, \ldots, m-1\}$, then for some $i<^{N} m, \phi^{-1}(i)$ is not a chain.

This will be accomplished by a diagonal argument. Let $P=N$ and $\left\langle P_{i}: i \in N\right\rangle$ be a recursive partition of $N$ into infinite recursive sets. If $x \in P_{i}, y \in P_{j}$, and $i<^{N} j$, then let $x<^{P} y$. Now for each $i \in N$ we shall define $<^{P}$ on $P_{i}$ so as to assure that (1) holds when $\phi=\phi_{i}$.

First we inductively partition $P_{i}$ into two recursive parts, $A_{i}$ and $B_{i}$. Suppose that we have $A_{i}^{p}$ and $B_{i}^{p}$, where $p \in P_{i}$. If for some $q \in A_{i}^{p}, \phi_{i}^{(p)}(q)$ is not defined or is not a member of $\{0, \ldots, m-1\}$, then put $p$ into $B_{i}$. Otherwise put $p$ into $A_{i}$. Notice that if $\phi_{i}$ satisfies the hypothesis of (1) then $A_{i}$ will be an infinite set.

If $p \in A_{i}$ and $q, r \in B_{i}$, where $q<^{N} r$, let $p<^{P} q<^{P} r$. We now finish our construction of $\left(P,<^{P}\right)$ by inductively defining $<^{P}$ on $A_{i}$. Suppose that we have $\left(A_{i},<^{P} \uparrow A_{i} \times A_{i}\right)^{p}$ and $p \in A_{i}$. Since $p \in A_{i}$ we have that $A_{i}^{p}$ is covered by $\phi_{i}^{-1}(0), \ldots, \phi_{i}^{-1}(m-1)$. Suppose $\left|A_{i}^{p}\right|=k$. Then this can be interpreted as the position at $X^{\prime}$ 's $(k+1)$ st turn in the game $G(m, n)$. Define $\left(A_{i},<^{P} \uparrow A_{i} \times A_{i}\right)^{p+1}$ 
according to the strategy $S$. Suppose the hypothesis of (1) holds for $\phi_{i}$. Then $A_{i}$ is infinite. Thus, since $S$ is a winning strategy for $G(m, n)$, the conclusion of (1) holds.

2.4. TheOREM. $5 \leqslant D^{\prime}(2)$. In particular, $5 \leqslant D(2)$.

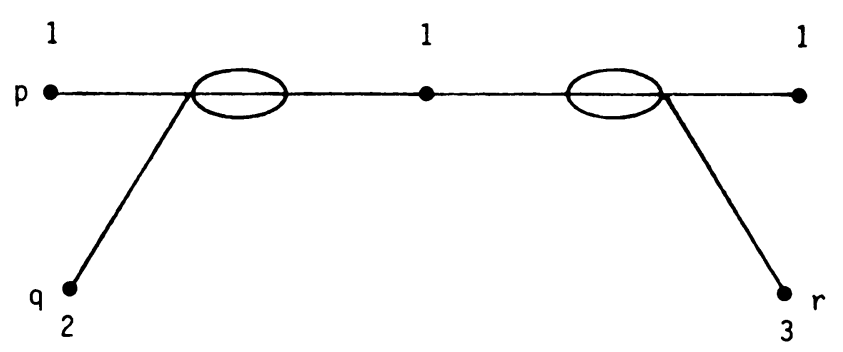

Proof. We must show that $X$ has a winning strategy for $G(4,2)$. We shall first isolate three winning positions for $X$. Then we shall show that $X$ can force $Y$ into one of these positions. For clarity it is necessary to use diagrams to describe various positions. Consider the diagram above. It illustrates a partial ordering, say $\left(P,<^{P}\right)$, that has been covered by sets. If two points are connected by lines then the point to the left is smaller than the point to the right. The loops represent possibly empty chains. A letter next to a point is its name; a number next to a point corresponds to the "color" $Y$ has given it. Thus $p \mid q$ and $p<^{P} r$. This position is called a 23 block. The prefix corresponds to the colors of $q$ and $r$. Several blocks may be connected to form a string. The diagram below illustrates a 2232 string. The 32 block of this string is called the third block. Similar definitions are made for other ordinals.

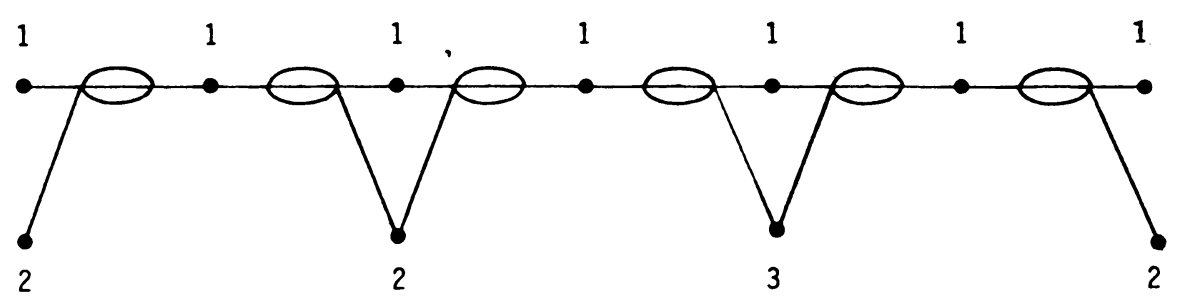

Each of our winning positions for $X$ will be certain strings. We now identify some useful plays that $X$ can make at a block in a string. Consider the figure below. Each of these plays has a name, for instance $A(p)$. This means we make play $A$ using the new element $p . p, q, r, s$ are made incomparable to any point outside their block to which they are not forced by transitivity to be comparable. Thus, if $A(p)$ and $B(q)$ are played in successive blocks of a string, $p \mid q$. However, if $C(r)$ and $D(s)$ are played in successive blocks of a string, $r<>s$. Notice that $Y$ cannot color $p$, $q, r$, or $s 1$. 

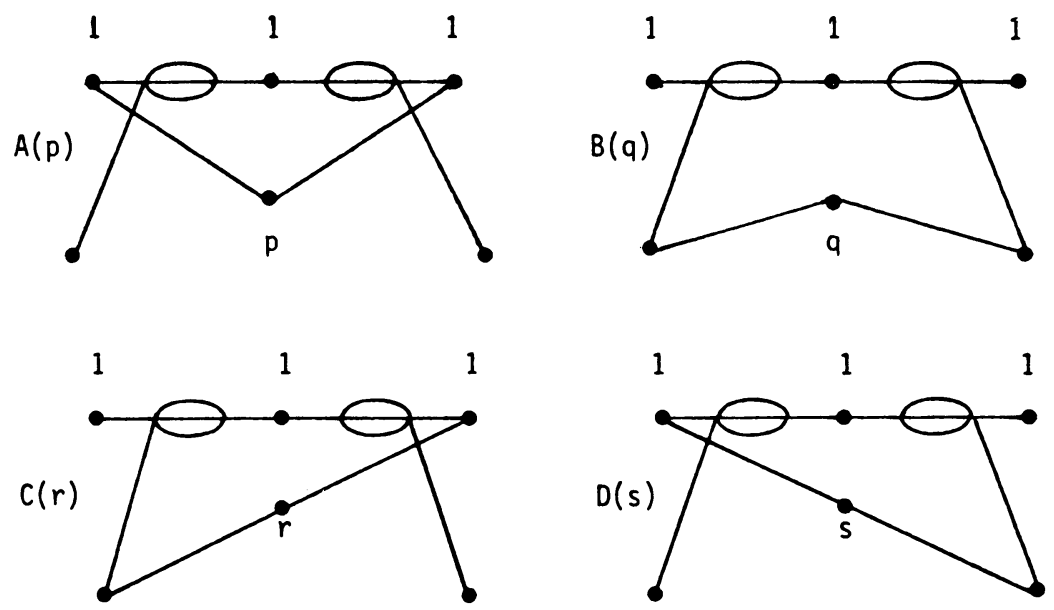

2.5. LeMMA. $A 232323$ string is a winning position for $X$.

Proof. We describe $X$ 's winning strategy. First $X$ plays $B(p)$ at 3 , i.e., $B(p)$ is played at the third block of the 232323 string. To avoid losing $Y$ must color $p 2,3$, or 4. Say $Y$ colors $p 2$. Then $X$ plays $D(p+1)$ at 4. Then $p \mid p+1 . Y$ must color $p+14$. Now $X$ plays $A(p+2)$ at 5 and $Y$ is helpless. If $Y$ colors $p 3$ then $X$ plays $C(p+1)$ at 2. Again $Y$ must color $p+14$. Now $Y$ is beaten when $X$ plays $A(p+2)$ at 1 . If $Y$ colors $p 4$ then $X$ plays $A(p+1)$ at 2 to win.

\subsection{LEMMA. $A 2232$ string is a winning position for $X$.}

Proof. Again we describe $X$ 's winning strategy. First $X$ plays $D(p)$ at 1 . If $Y$ colors $p 4$ then $X$ plays $A(p+1)$ at 2 and wins. So suppose $Y$ colors $p 3$. Then $X$ plays $D(p+1)$ at 2 . $Y$ is forced to color $p+14$. Thus $X$ wins by playing $A(p+2)$ at 3.

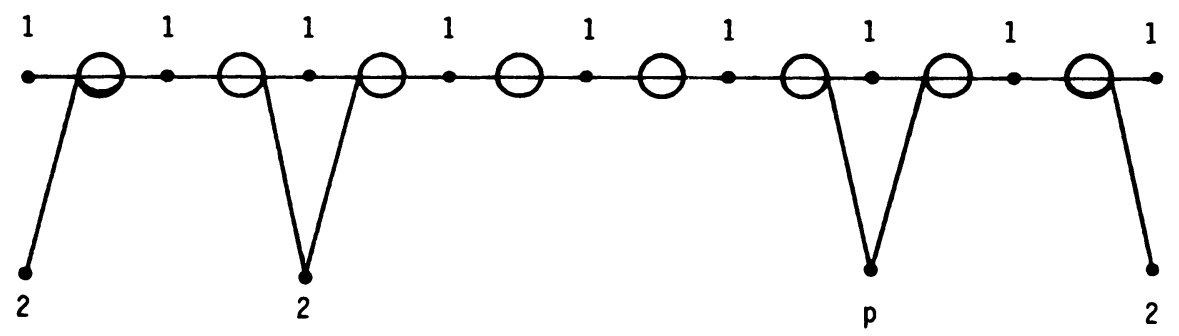

2.7. LEMMA. A 222 string is a winning position for $X$ if the last loop in the last block contains at least four elements that $Y$ has colored 1.

Proof. This time $X$ 's strategy is a little more complicated. $X$ 's first play is to create a new block by playing $p$ in the preceding diagram. If $Y$ colors $p 3$ then we have a 2232 string and we are done by 2.6. A similar thing holds if $Y$ colors $p$ 4. So suppose $Y$ colors $p 2$. Then we have a 2222 string. Now $X$ plays $D(p+1)$ at $1 . Y$ must color $p+13$ or 4 . Since either of these plays is equivalent, say $Y$ colors $p+1$ 3. Now $X$ plays $D(p+2)$ at 3 . $Y$ must color $p+23$ or 4 . If he colors $p+24$ he is finished after $D(p+3)$ at 2 . So suppose he colors $p+23$. $X$ replies by playing 
$p+3$ as diagrammed below. We now have a four block string. If $Y$ colors $p+32$ then $X$ wins by playing $D(p+4)$ at 2 and $D(p+5)$ at 3 . (Notice that $p+2$ is now in the fourth block.) If $Y$ colors $p+33$ then disregarding the last block we have a 2232 string. We are done by the proof of 2.6 since $D(p+1)$ was the recommended first move for $X$ on a 2232 string. The same argument holds if $Y$ colors $p+34$.

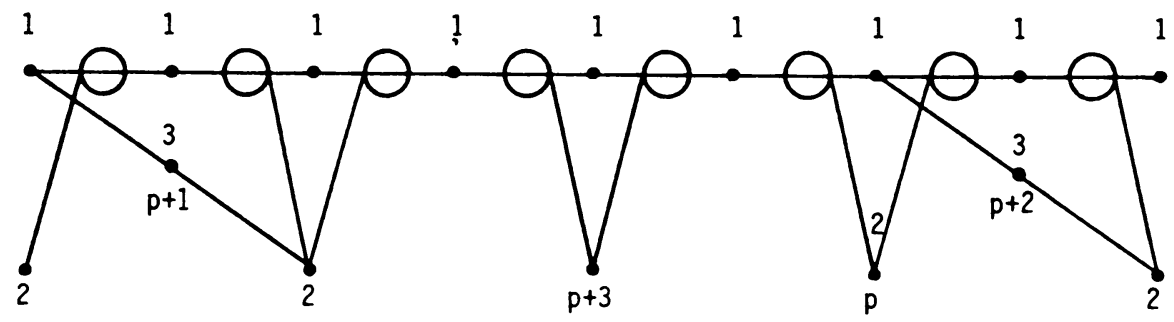

Of course, $x x x, x y x y x y, x x y x$, and $x y x x$ strings are also winning positions for $X$, where $x$ and $y$ are distinct members of $\{2,3,4\}$. Let $H$ be the following modification of $G(3,1)$. The rules are the same except that the game ends after each player has made nine plays and $X$ wins iff three consecutive elements of $X$ 's linear order are colored $x x x$, four consecutive elements are colored $x x y x$ or $x y x x$, or six consecutive elements are colored $x y x y x y$, where $x$ and $y$ are distinct colors.

2.8. LemMa. $X$ has a winning strategy for $H$.

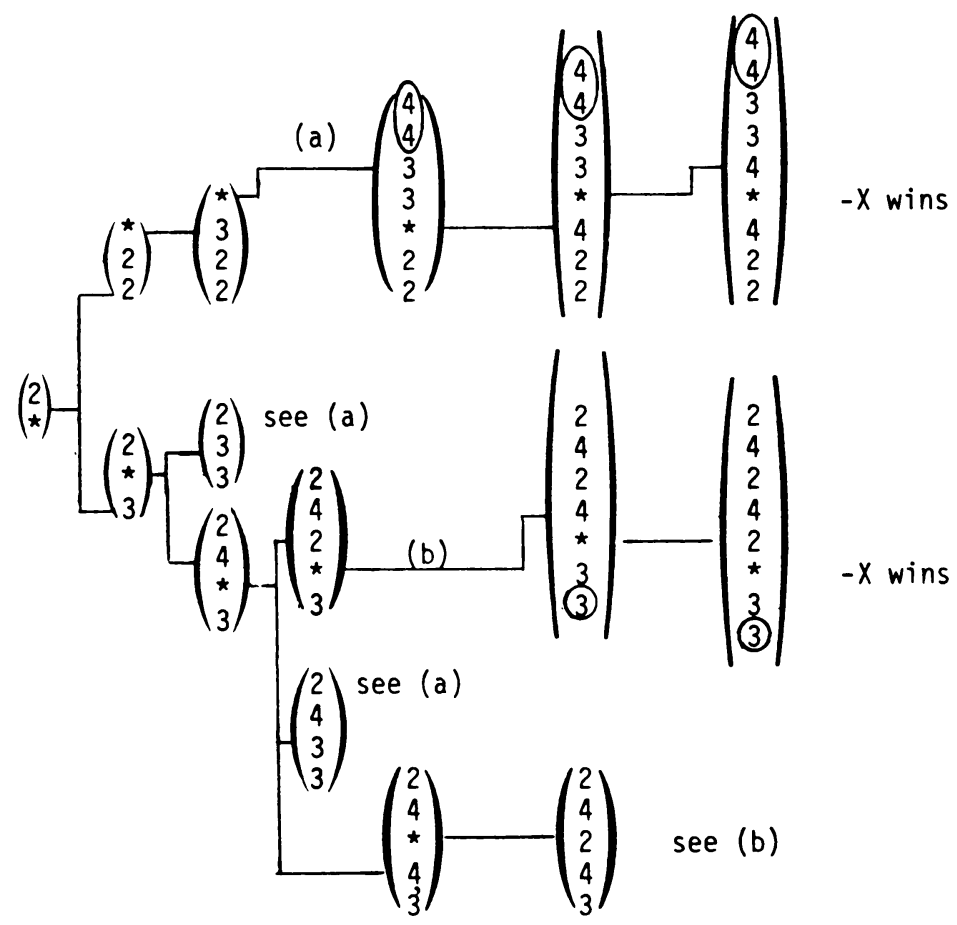

Proof. Say $Y$ uses the colors 2, 3, and 4. The details of $X$ 's strategy are left to the reader. The preceding diagram is provided as a guide. The sequences in parentheses represent the position at $Y$ 's turn. The * is $X$ 's last play, which $Y$ must 
now color. The branches represent $Y$ 's alternatives. We do not consider alternatives that immediately cause $Y$ to lose or that are alphabetic or symmetric variations. Steps have been left out following (a) and (b). This is because after (a) $Y$ may stall a little by using at most two 4's, but finally he must put a 3 in the desired position. These 4's are circled. A similar thing occurs after (b).

Now we finish the proof of the theorem. $X$ starts the game by building a linear ordering consisting of $4\left(6 \cdot 2^{9}-1\right)-3=12,281$ elements. $Y$ is forced to color $6 \cdot 2^{9}-1$ of these the same color, say 1 . Call these elements $L$. $X$ 's $12,281+i$ th play, for $0<<^{N} i \leqslant{ }^{N} 9$, will consist of choosing an element $a_{i}$ of $L$ and then introducing a new element $b_{i}$ which is incomparable to $a_{i}$, but comparable to all other previous elements. $a_{i}$ will be chosen so that there are at least $6 \cdot 2^{9-i}-1$ elements of $L$ above and below $a_{i}$ and also at least $6 \cdot 2^{9-i}-1$ elements of $L$ separating $a_{i}$ from any previous $a_{j}$. Since the previous $a_{j}$ also satisfy this condition, $a_{i}$ can be chosen from any interval of the previous $a_{j}$. The $b_{i}$ 's will form a chain. $Y$ will have to color each $b_{i} 2,3$, or 4 . Thus the position after $Y$ has made 12,290 plays will be a string of eight blocks. Since each $a_{i}$ will be separated from each other $a_{j}$ by at least $6 \cdot 2^{0}-1=5$ elements of $L$, the last loop in each block can be taken to have at least four elements that $Y$ has colored 1. By $2.8 X$ can play so that this string includes an $x x x, x x y x, x y x x$ or $x y x y x y$ string. Thus by $2.5-2.7, X$ has a winning strategy.

\subsection{Corollary. $D^{\prime}(n)+4 \leqslant D^{\prime}(n+1)$. In particular, $4 n-3 \leqslant D(n)$.}

Proof. We make two simple modifications to the proof of Theorem 2.4. The elements in blocks that were colored 1 will be replaced by finite, width $n$ partial orderings such that for each of the first $D^{\prime}(n)$ colors, $i, Y$ has colored some member of the partial ordering $i$. The following diagram shows one of these new blocks.

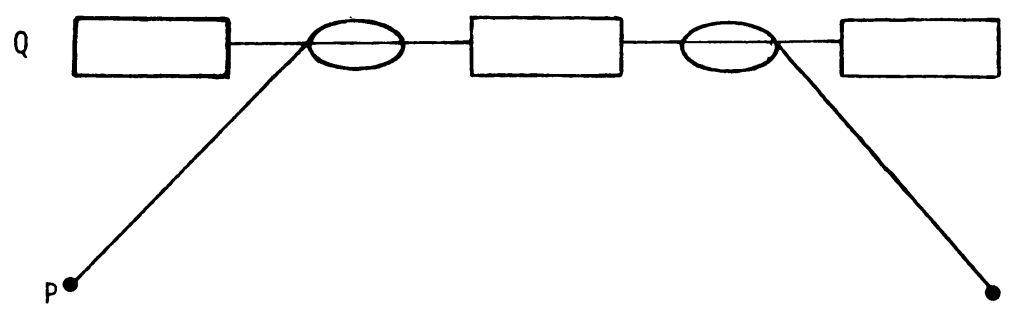

The rectangles are the new partial orderings that replaced the elements colored 1. The circles are now chains of width $n$ partial orderings. Note that if $q \in Q$ then $p \mid q$. Thus $Y$ cannot color $p$ any of the first $D^{\prime}(n)$ colors. Now 2.5-2.7 hold with this new kind of block and with $G(4,2)$ replaced by $G\left(D^{\prime}(n)+3, n+1\right)$. The other modification is that we start the game by constructing a chain of

$$
\left(\begin{array}{c}
D^{\prime}(n)+3 \\
D^{\prime}(n)
\end{array}\right)\left(6 \cdot 2^{9}-1\right)
$$

rectangles in linear order. The $a_{i}$ in the proof of Theorem 2.4 are now rectangles. 
3. Uniformity. In this section we shall show that for any $n \in N$ there exists an effective procedure for covering any recursive width $n$ partial ordering with $D(n)$ recursive chains. This is not to say that we can find this procedure. If $\phi_{e}$ is a decision procedure [on $A$ ] for a width $n$ partial ordering, call that partial ordering $\left(P_{e},<^{e}\right)\left[\left(P_{e}^{A},<^{e, A}\right)\right]$.

3.1. THEOREM. There exists a partial recursive function $f: N \times N \rightarrow N$ such that if $\phi_{e}$ is a decision procedure for a width $n$ partial ordering then $\left\{\left\{x \in P_{e}: f(e, x)=i\right\}\right.$ : $i<D(n)\}$ is a covering of $P_{e}$ by $D(n)$ chains.

Proof. We shall construct a recursive width $n$ partial ordering $\left(U,<^{U}\right)$ and a partial recursive function $g: N \times N \rightarrow N$ so that (1) below holds, where $g_{e}$ is defined by $g_{e}(x)=g(e, x)$.

(1) If $\phi_{e}$ is a decision procedure for a width $n$ partial ordering then $g_{e} \uparrow P_{e}$ is an order preserving embedding of $\left(P_{e},<^{e}\right)$ into $\left(U,<^{U}\right)$.

Since $\left(U,<^{U}\right)$ will be recursive and have width $n$, there will exist disjoint recursive chains $C_{1}, \ldots, C_{D(n)}$ which cover $U$. Then $f$ will be defined by:

$$
f(e, x)= \begin{cases}i, \quad \text { where } g(e, x) \in C_{i}, & \text { if } g(e, x) \text { is defined, } \\ \text { undefined, } & \text { if } g(e, x) \text { is undefined. }\end{cases}
$$

Clearly this $f$ will suffice.

Thus it only remains to construct $\left(U,<^{U}\right)$ and $g$. Let $U=N$ and let $\left\langle U_{e}\right.$ : $e \in N>$ be a recursive partition of $N$ into infinitely many infinite sets. We first define $g$ by defining $g_{e}^{s}$ by induction on $s$. During this process we will also inductively partition $U_{e}$ into $A_{e}$ and $B_{e}$. Suppose we have $g_{e}^{s}, A_{e}^{s}$, and $B_{e}^{s}$. If $s \notin U_{e}$ then $g_{e}^{s}=g_{e}^{s+1}, A_{e}^{s}=A_{e}^{s+1}$, and $B_{e}^{s}=B_{e}^{s+1}$. Suppose $s \in U_{e}$. Let

$$
p_{s}=<^{N}-\min \left(N \backslash d_{0}\left(g_{e}^{s}\right)\right) \text {. }
$$

If $\phi_{e}^{(s+1)}$ is not a decision procedure on $E=d_{0}\left(g_{e}^{s}\right) \cup\left\{p_{s}\right\}$ for a width $n$ partial ordering, then put $s$ into $B_{e}$ and let $g_{e}^{s+1}\left(p_{s}\right)$ be undefined. Otherwise let $g^{s+1}\left(p_{s}\right)=$ $s$. If $p_{s} \in P_{e}^{E}$ put $s$ into $A_{e}$. Otherwise put $s$ into $B_{e}$. Notice that if the hypothesis of (1) holds then $g_{e} \mid P_{e}$ is a one-to-one map onto $A_{e}$. Finally we define $<^{U}$. For $x, y \in U$ let $x<^{U} y$ iff one of the following conditions holds.

(i) $x \in U_{i}, y \in U_{j}$ and $i<^{N} j$.

(ii) $x \in A_{e}$ and $y \in B_{e}$.

(iii) $x, y \in B_{e}$ and $x<^{N} y$.

(iv) $x, y \in A_{e}$ and $g_{e}^{-1}(x)<^{e, E} g_{e}^{-1}(y)$, where $s=<^{N}-\max (x, y)$ and $E=d_{0}\left(g_{e}^{s}\right)$ $\cup\left\{p_{s}\right\}$.

By the construction $\left(U,<^{U}\right)$ is a recursive width 2 partial ordering and (1) holds.

4. Open questions. Theorem 1.1 shows that $D$ is a well-defined function. Theorem 2.4 and Corollary 2.9 show that $D$ is far from the identity. An obvious question is what is $D(2)$ ? More generally, for each $n \in N$, what is $D(n)$ ? The latter question may be unanswerable since $D$ may not be recursive. One way of showing that $D$ is recursive would be to show that $D=D^{\prime}$ and that $D^{\prime}$ is recursive. We know that $D^{\prime} \leqslant D$. The reason that $D^{\prime}$ might be strictly less than $D$ is that $Y$ 's winning strategy for $G\left(n, D^{\prime}(n)\right)$ might not be recursive. Let the game $G_{k}(m, n)$ have the 
same rules as $G(m, n)$ except that it ends after each player has made $k$ plays. Let $D_{k}(n)$ be the least natural number $i$ such that $Y$ has a winning strategy for $G_{k}(m, i)$. It is easy to see that $D_{1}, D_{2}, \ldots$ is a nondecreasing sequence of recursive functions such that $\lim _{k \rightarrow \infty} D_{k}=D^{\prime}$. Thus $D^{\prime}$ is recursive in $O^{\prime}$. One might show that $D^{\prime}$ was recursive by showing that there was a recursive function $f$ such that $D(n)=$ $D_{f(n)}(n)$. These considerations lead to the following questions.

4.1. Questions. Are $D$ and $D^{\prime}$ recursive? If not, in what degrees are they recursive?

4.2. Question. Is $D^{\prime}$ equal to $D$ ?

Another approach to analyzing $D$ would be to study its rate of growth. Theorem 1.1 shows that $D$ is bounded by an exponential function. Using a modification of the proof of 2.9 it is not difficult to see that no linear function can be an upper bound for $D$.

\section{REFERENCES}

[B] D. Bean, Effective coloration, J. Symbolic Logic 41 (1976), 469-480.

[B1] __ Recursive Euler and Hamilton paths, Proc. Amer. Math. Soc. 55 (1976), 385-394.

[D] R. P. Dilworth, A decomposition theorem for partially ordered sets, Ann. of Math. (2) 51 (1950), 161-166.

[J] C. Jockush, Ramsey's theorem and recursion theory, J. Symbolic Logic 37 (1972), 268-279.

[K] H. Kierstead, Recursive colorings of highly recursive graphs (in preparation).

[MN] G. Metakides and A. Nerode, Recursion theory and algebra Algebra and Logic, Lecture Notes in Math., vol. 450, Springer-Verlag, Berlin and New York, 1975, pp. 209-219.

[P] M. A. Perles, A proof of Dilworth's decomposition theorem for partially ordered sets, Israel J. Math. 1 (1963), 105-107.

[R] H. Rogers, Theory of recursive functions and effective compatability, McGraw-Hill, New York, 1967.

[S] J. H. Schmerl, Recursive colorings of graphs, Canad. J. Math. 32 (1980), 821-830.

[S1] , The effective version of Brook's theorem (in preparation).

Department of Mathematics, University of California, San Diego, California 92037

Current address: Department of Mathematics, University of South Carolina, Columbia, South Carolina 29208 